\title{
Enseñanza de la bioética y planes de estudios basados en competencias
}

\author{
A. Couceiro-Vidal
}

\begin{abstract}
Cada vez más se acepta que la bioética debe formar parte de la formación de los profesionales de la medicina. No obstante, permanecen muchos puntos confusos, como su carácter de ética aplicada, o su diferencia con el profesionalismo, con la deontología profesional o con el derecho. En este artículo se trata de mostrar que la bioética es ética cívica aplicada a la relación clínica, lo que supone la incorporación de los derechos civiles y sociales a dicha relación, y la identificación y el manejo de los conflictos de valores surgidos de esta interacción. La enseñanza de la bioética en las facultades de medicina debe insertarse, como el resto de las asignaturas, en el marco de las competencias. Definir conocimientos y desarrollar habilidades concretas y específicas es también el objetivo de esta disciplina. En el presente artículo se presenta una propuesta de competencias y contenidos para la enseñanza de la bioética, articulada en los dos niveles (preclínico y clínico) que caracterizan la enseñanza de la medicina.
\end{abstract}

Palabras clave. Bioética. Educación médica. Enseñanza por competencias. Humanidades médicas.

\section{Teaching bioethics in outcome-based curricula}

Increasingly accepted is that the bioethics must be part of the training of professionals of medicine, yet many points remain unclear, as its character of ethics applied, or its dispute with the professionalism, with the deontology professional, or with the law. This article tries to show that the bioethics civic ethics is applied to the relationship clinic, which represents the incorporation of civil and social rights this relationship, and the identification and management of conflicts of values arising from this interaction. The teaching of bioethics in the faculties of medicine should be inserted, like the rest of the subjects, in the framework of competences. Define knowledge and develop specific skills, is also the objective of this discipline. This article presents a proposal of competence and content for the teaching of bioethics, articulated in the two levels (preclinical and aged) that characterized the teaching of medicine.

Key words. Bioethics. Educational competences. Medical education. Medical humanities.

\section{La bioética, una nueva disciplina académica}

Las facultades de medicina, consecuentes con su responsabilidad social, tienen que formar el tipo de profesional que la sociedad necesita. Sus graduados deben poseer las características esenciales de la profesión, pero también conocer las características específicas de su entorno cultural, cívico y social. Todo ello perfila algunos de los problemas de la tipificación de la bioética, una nueva disciplina del currículo de medicina.

Por un lado, es el propio entorno cultural y social el que introduce graves confusiones sobre lo que es la bioética. Pero, además, a diferencia de lo que algunos autores argumentan, su enseñanza en las facultades de medicina debe insertarse en la misma lógica que la del resto de las materias, en el marco del currículo basado en competencias, y utilizando como metodología principal el aprendizaje basado en problemas, sistematizado desde finales de los años sesenta por la facultad de McMaster, en Hamilton, Ontario, Canadá [1].

Esta disciplina tiene su origen en la cultura norteamericana, en la que el principio de 'libertad moral' rige la vida política desde el siglo xVIII, y se aplica tanto en el orden religioso (principio de libertad religiosa) como en el político (principio de democracia). El punto de partida es que todo ser humano es un agente moral
Departamento de Psiquiatría. Facultad de Medicina. Universidad Autónoma de Madrid. Madrid, España.

Correspondencia Dra. Azucena Couceiro Vidal. Departamento de Psiquiatría. Facultad de Medicina. Universidad Autónoma de Madrid. Arzobispo Morcillo, s/n. E-28029 Madrid.

E-mail

acouceiro@arrakis.es

Trabajo realizado dentro del proyecto de investigación ref. HUM2005-02105/FISO, financiado por el Ministerio de Educación y Ciencia, con el título 'Racionalidad axiológica de la práctica tecnocientífica'.

\section{Agradecimientos A A. Oriol Bosch y H. Pardell. Sus publicaciones sobre la enseñanza de la medicina y el profesionalismo médico han sido de gran ayuda para delimitar la relación entre profesionalismo y bioética.}


autónomo, y que por ello debe ser respetado por los que mantengan posiciones morales distintas. Ninguna moral puede imponerse a las personas en contra de su conciencia. De aquí que la ética civil y social no se pueda construir sólo por un grupo determinado, sino que debe ser el resultado del consenso deliberativo logrado por todos los ciudadanos, por todos los agentes morales, mediante las reglas propias del sistema democrático. Aplicar todos estos supuestos a la relación clínica es, precisamente, lo que genera la aparición de la bioética.

Las sociedades en cuya vida civil cotidiana no se tienen tan claros estos presupuestos tienden a confundir la bioética con la religión, con la deontología profesional o con el derecho. La primera confusión es un error tan grave como frecuente, que, entre otros, nos conduciría a negar el respeto a un derecho descubierto en la Modernidad y plasmado en las constituciones de nuestros países, el derecho a la libertad de conciencia.

La segunda confusión deriva de entender que hay un único tipo de valores que se deben tener en cuenta en la relación clínica, aquéllos que han dado sentido a la profesión durante siglos, que constituyen nuestra 'tradición profesional', y que se han ido plasmando en los códigos deontológicos. Esta afirmación se ha podido mantener en el seno de sociedades jerárquicas, no democráticas, en las que la única forma posible de entender la relación médico-paciente ha sido el modelo paternalista, pero con la introducción de los regímenes democráticos, las modificaciones, también para la relación clínica, son profundas, y generan conflictos de valores inéditos hasta ahora en nuestra tradición [2].

Sociedad plural es aquélla en la que los ciudadanos comparten unos mínimos morales que les permiten tener una base común para ir construyendo el mundo que les circunda. Tales mínimos pueden concretarse en el respeto a los derechos humanos de la primera, segunda y tercera generaciones o, lo que es lo mismo, en los valores de libertad, igualdad y solidaridad. Estos mínimos éticos se plasman en las constituciones de cada país y, entre otras, en aquellas normas legislativas que desarrollan los derechos constitucionales, de forma que todos los ciudadanos estamos obligados a cumplirlas, porque, además, creemos que es lo mejor y más justo para nuestra sociedad [3]. Por eso derechos como el de la libertad de conciencia o el derecho a la asistencia sanitaria también son vinculantes en la relación clínica, e introducen nuevos valores, como la autonomía del paciente o la justicia distributiva aplicada a los recursos sanitarios, que deben tenerse en cuenta $[4,5]$.

Los profesionales, como ciudadanos que somos, también estamos obligados a su cumplimiento. En la actualidad, no se puede concebir una relación clínica al margen de los valores básicos que constituyen el fundamento de la ética cívica. Este cambio social tan importante ha modificado también el concepto de profesión, que debe ejercerse en un entorno muy diferente, caracterizado por un individualismo extremo, con acceso fácil a la información especializada, muy inestable en sus condiciones sociales, y burocratizado a través de todas sus instituciones [6]. Aparece así un nuevo concepto, el de 'profesionalismo', que puede definirse como el conjunto de principios y compromisos para mejorar los resultados en salud del paciente y maximizar su autonomía, creando relaciones caracterizadas por la integridad, la práctica ética, la justicia social y el trabajo en equipo [7,8].

Es importante retomar esta idea, porque, si bien la bioética y el profesionalismo pueden entenderse como el resultado de tantos cambios sociales acaecidos, no son disciplinas superponibles. La bioética es un tipo de ética aplicada, que presupone la introducción en la medicina de los valores democráticos que fundamentan la vida civil de las sociedades occidentales, y que capacita al alumno para fundamentar juicios morales -elementos que se deben considerar, procedimiento, metodología, etc.- cuando en el ejercicio de su profesión se encuentre ante conflictos éticos entre esos mismos valores $[9,10]$. El profesionalismo surge de la reflexión de los profesionales ante los cambios no sólo de valores, sino institucionales, laborales, etc., que afectan a la relación clínica, y que conducen a un replanteamiento del contrato social entre la profesión médica y el Estado [11].

Dicho de otra manera, la bioética irrumpe en la relación clínica de la mano de la sociedad, que, por un parte, exige el respeto a la autonomía del ciudadano, incluso cuando está enfermo, y, por otra, entiende que el acceso a la asistencia sanitaria es una cuestión de justicia distributiva. Es un tipo de ética aplicada, con un método propio, un marco de valores cívicos que se han de tener en cuenta, y unos valores propios de la actividad a la 
que se aplica [12]. El profesionalismo constituye la respuesta actual de los profesionales de la medicina, que buscan la manera de no perder en el nuevo y complejo entorno social valores fundamentales para el ejercicio de la profesión médica.

Existe una relación muy estrecha entre el nuevo profesionalismo y la bioética. Esta última ha sido un elemento clave, que ha ayudado a los profesionales sanitarios a redefinir su papel y a generar un nuevo tipo de ética profesional que ha acogido la mayoría de los postulados de la bioética. Ahora bien, el nuevo profesionalismo bebe de la bioética, pero no la agota. La bioética no puede reducirse a ética profesional. No hay duda de que el alumno tiene que conocer y asumir las obligaciones que comporta formar parte de un cuerpo profesional, pero éste es otro aspecto de su formación que debería impartirse al final de la carrera, cuando está finalizando el proceso formativo y es inminente su incorporación a la profesión médica $[13,14]$.

La bioética es una nueva disciplina académica que ha sido, de entre todas las humanidades médicas, la que más se ha desarrollado, y probablemente la que exigen con gran urgencia los planes de estudios de medicina para dotar a sus alumnos de las competencias que les permitan manejar los conflictos de valores de una relación clínica auténticamente democrática [15-17].

\section{Enseñanza de la bioética en el marco de las competencias}

A partir de los años setenta, los expertos en educación médica comienzan a recomendar un nuevo enfoque del aprendizaje, un marco que permita al alumno adquirir no sólo contenidos teóricos, sino también una capacidad reflexiva y evaluativa de las situaciones que tendrá que resolver en el ámbito de su profesión. Este complejo aprendizaje requiere la inmersión en contextos concretos. Hay que organizarlo de manera que se produzca en las situaciones de trabajo lo más reales posibles, lo que se logra mucho mejor en el marco de un currículo basado en competencias, y utilizando preferentemente la metodología educativa del aprendizaje basado en problemas [18].

Si bien es cierto que no se han demostrado diferencias significativas entre el modelo basado en competencias y el tradicional, también lo es que el aprendizaje basado en problemas presenta claras ventajas cuando se miden competencias transversales, a las que tradicionalmente no se les ha prestado atención, y que son fundamentales para desarrollar cualquier profesión [19].

Se entiende por competencias el conjunto de conocimientos (conocer y comprender), habilidades (saber cómo actuar) y actitudes humanas que permiten una excelente práctica médica, adecuada al contexto social en el que se desarrolla [20]. La competencia determina el grado de capacidad operativa del individuo en un entorno determinado [21]. Presupone una base cognitiva que incluye conocimientos, habilidades y actitudes. Las competencias se pueden definir de modo amplio (por ejemplo, comunicación efectiva) o concreto (por ejemplo, manejo del paciente diabético no complicado), si bien estas últimas son más fáciles de evaluar. Pero lo importante a la hora de definir una competencia es que cumpla las siguientes características: relevancia en el entorno profesional, transferibilidad al estudiante y posibilidad de ser evaluada objetivamente.

Conocimientos, habilidades y actitudes son, pues, los tres componentes claves de todo proceso de aprendizaje. La enseñanza de la bioética a los estudiantes de medicina no puede ser ajena ni al paradigma educativo de las competencias, ni al desarrollo de sus elementos. En bioética existe un conjunto amplísimo de conocimientos que constituyen el cuerpo de la disciplina, que se pueden enseñar mediante las clases teóricas. Estos conocimientos no son mera especulación sin relevancia práctica, sino que constituyen el andamiaje básico que hace posible la adquisición de habilidades. El alumno logrará así las competencias para actuar sobre los conflictos éticos que surgen en la vida diaria de las profesiones sanitarias, y también para analizarlos críticamente y tomar decisiones que sean éticamente consistentes.

Es claro que la adquisición de competencias constituye el objetivo inmediato de todo proceso formativo en esta materia, pero mal se pueden desarrollar las habilidades que le son inherentes si no existe un mínimo de conocimientos. Ahora bien, tampoco sirve de mucho que un alumno conozca la teoría de la bioética si no sabe cómo aplicarla críticamente para analizar un caso clínico. Vale decir que los dos niveles son complementarios, pero también que presentan sus diferencias, pues no es lo mismo saber, en teoría, 
cuáles son los elementos del consentimiento informado, que tener la destreza práctica para llevar a cabo este proceso con cada paciente. Hay, pues, un objetivo de conocimientos y otro de adquisición de habilidades, que deben plantearse conjuntamente en la formación del alumno.

Cabe preguntarse también si la bioética puede o debe inducir un cambio de actitudes. La mayor parte de los autores ha contestado a esta cuestión negativamente, y no sólo debido al peligro de intentar 'adoctrinar' o manipular a las personas, sino también porque las actitudes fundamentales, o el carácter moral de los estudiantes de medicina, ya está formado cuando entra en la universidad. Así lo destaca, entre otros, un trabajo ya clásico sobre la enseñanza de la bioética, firmado por nueve de los más representativos bioeticistas estadounidenses [22].

Durante el período de formación en medicina, los programas de enseñanza de la bioética tienen que enseñar conocimientos y desarrollar habilidades, pero no directamente actitudes. La realidad, a diferencia de la tesis mantenida por los autores estadounidenses, es que un proceso formativo en bioética también transforma las actitudes y el carácter moral de las personas. Ahora bien, esa modificación es secundaria al propio proceso de aprendizaje de conocimientos y habilidades, y no directamente inducida. El solo hecho de ser capaz de identificar los conflictos éticos de la práctica clínica, y de poder dar respuestas racionales y prudentes, conduce a que el alumno interiorice un procedimiento, una forma de actuación y una sensibilización ante los valores implicados en la relación clínica.

A modo de ejemplo, si el alumno conoce la teoría del consentimiento informado -origen, fundamento y elementos- y se ha formado en la habilidad comunicativa de la entrevista clínica y la transmisión correcta de la información, interiorizará una actitud de receptividad ante este proceso. Sólo de esta manera, capacitado para responder a este derecho del paciente, superará la 'burocratización' del proceso, que lo reduce a la firma de un documento, muchas veces con un contenido que nadie le ha explicado al paciente, y que por ello carece de validez, tanto ética como jurídica. Se obtiene así un cambio, ya que la adquisición progresiva de competencias conduce a una mayor responsabilidad. Y es que todo programa docente en bioética acaba girando en torno al concepto de profesional responsable y capacitado.
Tabla I. Valores profesionales, actitudes, comportamientos y ética (Conferencia Nacional de Decanos de Facultades de Medicina Españolas. Libro blanco de la titulación en medicina).

- Reconocer los elementos esenciales de la profesión médica, incluyendo los principios éticos y las responsabilidades legales

- Comprender la importancia de tales principios para el beneficio del paciente, de la sociedad y la profesión, con especial atención al secreto profesional

- Saber aplicar el principio de justicia social a la práctica profesional

- Desarrollar la práctica profesional con respeto a la autonomía del paciente, a sus creencias y cultura

- Reconocer las propias limitaciones y la necesidad de mantener y actualizar su competencia profesional

- Desarrollar la práctica profesional con respeto a otros profesionales de la salud

Tabla Il. Ética médica (Agencia Nacional de Evaluación de la Calidad, ANECA, 2005).

- Los principios éticos y deontológicos del ejercicio de la medicina

- Normativa nacional e internacional. Código deontológico de la Organización Médica Colegial

- Principales situaciones conflictivas en bioética. Ética en investigación

- Secreto profesional

- La objeción de conciencia por motivos profesionales

- Intrusismo. Publicidad

- Los derechos y deberes de los pacientes

- El diagnóstico médico: información, limitaciones. Ética en la relación clínica

- Equidad en la distribución de los recursos y la protección de la salud pública

Una docencia con esta orientación es el mejor antídoto frente a dos extremos. Uno, el de adoctrinar, manipular o imponer; el otro, el de reducir esta formación a una mera información. Educar las actitudes mediante los conocimientos y las habilidades no sólo es posible, sino que constituye la manera más correcta de orientar el aprendizaje de la bioética en personas adultas [23]. También es la forma de no descuidar el llamado 'currículo informal', que se refiere al proceso de transformación individual que tiene 
Tabla III. Valores profesionales, actitudes, comportamientos y ética (Agencia para la Calidad del Sistema Universitario en Cataluña. Competencias esenciales para los licenciados en medicina en Cataluña).

- Reconocimiento de los elementos esenciales de la profesión médica, incluyendo los principios morales y éticos y las responsabilidades legales subyacentes a la profesión

- Valores profesionales, que incluyen la excelencia, el altruismo, el sentido del deber, la compasión, la empatía, la responsabilidad, la honradez, la integridad y el compromiso con el método científico

- Asunción del propio desarrollo profesional y el mantenimiento de la competencia profesional

- Comprensión del hecho de que cada médico tiene la obligación de promocionar, proteger y mejorar estos elementos para el beneficio de los pacientes, de la profesión y de la sociedad en general

- Reconocimiento de que una buena práctica médica depende de la comprensión mutua y de la relación entre el médico, el paciente, los familiares y la comunidad; y también del respeto al bienestar del paciente, a la diversidad cultural, a las creencias y a su autonomía

- Habilidad para aplicar los principios de razonamiento moral y de toma de decisiones en conflictos con y entre elementos de tipo ético, legal y profesional, incluyendo aquéllos que se deben a restricciones de carácter económico, a la comercialización de los cuidados de salud y a los avances científicos

- Autoevaluación y reconocimiento de la necesidad de la mejora personal continua, conociendo las propias limitaciones; también las referidas al conocimiento médico

- Respeto por todos los profesionales de la salud y habilidad para promocionar una relación positiva y de colaboración entre ellos

- Reconocimiento de la obligación de proporcionar tratamiento a los enfermos terminales, incluyendo la atenuación de síntomas

- Reconocimiento de los aspectos éticos, legales y técnicos en la documentación del paciente, en el plagio, la confidencialidad y la propiedad intelectual

- Habilidad para planear y gestionar eficazmente el tiempo propio y las actividades, para afrontar la incertidumbre, y habilidad para adaptarse al cambio

- Responsabilidad personal para el cuidado y la asistencia a los pacientes

- Contribución al progreso de la medicina, con una actitud permanente de investigación incorporada a la actividad clínica

lugar como consecuencia de las situaciones e interacciones humanas no estrictamente académicas, y que es el que parece tener un mayor poder condicionador de las conductas [24].

Es notorio que los alumnos pueden sufrir una importante transformación negativa a su paso por la carrera de medicina. La experiencia humana y docente puede no sólo potenciar, sino inhibir el desarrollo moral de los estudiantes [25]. La razón es que perciben una clara distancia entre lo que se les comunica verbalmente como los 'valores adecuados' y los ejemplos profesionales que observan en el campo clínico. Las actitudes, conductas y comentarios que perciben les revelan los valores de fondo que, en realidad, aplican los profesionales en ejercicio. La creación de hábitos reflexivos y la adquisición de competencias en bioética debería ser la forma mediante la cual las instituciones docentes estimulen un desarrollo moral positivo.

Pero ¿cuáles son las competencias a conseguir en esta disciplina? Tanto instituciones internacionales como nacionales las han señalado en sus documentos oficiales [26-28]. Si hacemos un somero análisis de las diversas propuestas aparecidas en nuestro país, podremos observar dos graves problemas. El primero, que son competencias demasiado amplias, a las que con excesiva frecuencia se suele tender en el ámbito de los valores, a diferencia de lo que ocurre en otras áreas competenciales del currículo médico (Tablas I, II y III). Por ello, no parecen muy adecuadas, pues, aunque cumplan con una de las características -relevancia profesional-, no lo hacen respecto de las dos restantes: ser transferible al estudiante, y posibilidad de ser evaluada objetivamente. Deberían ser mucho más concretas, y priorizar los aspectos más relevantes para el ejercicio profesional de un médico general, que es el 'producto' de una facultad de medicina, y no los de un especialista, que requerirá otro nivel de formación en bioética, centrado ya en los problemas concretos de su especialidad.

El segundo problema hace referencia a la mezcla entre los contenidos del profesionalismo y los de la bioética, lo que puede crear cierta confusión. Por ejemplo, cuando la Agencia Nacional 
Tabla IV. Aspectos que mejorarían el método.

\begin{tabular}{|c|c|c|}
\hline Competencias & Conocimientos (saber) & Habilidades (saber hacer) \\
\hline $\begin{array}{l}\text { Identificar los } \\
\text { aspectos éticos } \\
\text { de la relación clínica }\end{array}$ & $\begin{array}{l}\text { Juicios morales } \\
\text { Valores, principios, derechos } \\
\text { Principios de la bioética } \\
\text { Concepto de ética cívica } \\
\text { Bioética, deontología profesional y derecho }\end{array}$ & $\begin{array}{l}\text { Diferenciar entre conflictos morales y legales } \\
\text { Identificar y aplicar los derechos } \\
\text { constitucionales a la relación clínica }\end{array}$ \\
\hline $\begin{array}{l}\text { Realizar un proceso } \\
\text { de consentimiento } \\
\text { (o rechazo) válido } \\
\text { con el paciente }\end{array}$ & $\begin{array}{l}\text { Derechos de los pacientes } \\
\text { Elementos del consentimiento informado } \\
\text { Modelos de relación clínica y participación } \\
\text { del enfermo en la toma de decisiones }\end{array}$ & $\begin{array}{l}\text { Informar adecuadamente a un paciente } \\
\text { Comunicar malas noticias } \\
\text { Evaluar la competencia de un paciente }\end{array}$ \\
\hline $\begin{array}{l}\text { Saber cómo proceder } \\
\text { ante un rechazo de } \\
\text { tratamiento }\end{array}$ & $\begin{array}{l}\text { Derecho del paciente a rechazar tratamientos: } \\
\text { fundamento y contenido }\end{array}$ & $\begin{array}{l}\text { Evaluar: información que tiene el paciente, } \\
\text { voluntariedad, capacidad y autenticidad } \\
\text { de la decisión } \\
\text { Proporcionar alternativas sin lesionar } \\
\text { la voluntariedad }\end{array}$ \\
\hline $\begin{array}{l}\text { Saber cómo proceder } \\
\text { ante un paciente } \\
\text { incompetente }\end{array}$ & $\begin{array}{l}\text { Decisiones de representación } \\
\text { Planificación anticipada de las decisiones } \\
\text { Criterios para la toma de decisiones en niños } \\
\text { Criterios para la toma de decisiones } \\
\text { en adolescentes } \\
\text { Testamentos vitales y directivas anticipadas }\end{array}$ & $\begin{array}{l}\text { Informar y hacer participar a un paciente } \\
\text { parcialmente incompetente } \\
\text { Identificar al sustituto de un paciente } \\
\text { incompetente } \\
\text { Aplicar una directiva anticipada }\end{array}$ \\
\hline $\begin{array}{l}\text { Saber utilizar } \\
\text { racionalmente la } \\
\text { tecnología médica }\end{array}$ & $\begin{array}{l}\text { Tecnología como medio, no como fin } \\
\text { Concepto de limitación del esfuerzo } \\
\text { terapéutico (LET) } \\
\text { La LET en las enfermedades críticas y en } \\
\text { enfermedades crónicas: fundamento y praxis }\end{array}$ & $\begin{array}{l}\text { Realizar un juicio clínico que fundamente la } \\
\text { indicación de limitar el esfuerzo terapéutico }\end{array}$ \\
\hline $\begin{array}{l}\text { Manejar los } \\
\text { aspectos éticos de la } \\
\text { enfermedad terminal }\end{array}$ & $\begin{array}{l}\text { Conceptos de terminalidad y enfermedad } \\
\text { avanzada } \\
\text { Proceso de consentimiento informado } \\
\text { con el enfermo terminal } \\
\text { Sedación del enfermo terminal }\end{array}$ & $\begin{array}{l}\text { Establecer procesos comunicativos con } \\
\text { el enfermo terminal y su unidad familiar } \\
\text { Identificar los conflictos éticos más } \\
\text { frecuentes: uso de analgésicos potentes, } \\
\text { rechazo de tratamiento, nutrición } \\
\text { e hidratación artificial, etc. }\end{array}$ \\
\hline $\begin{array}{l}\text { Manejar de forma } \\
\text { adecuada los datos } \\
\text { de la historia clínica }\end{array}$ & $\begin{array}{l}\text { Secreto médico frente a confidencialidad } \\
\text { Datos clínicos, información sensible } \\
\text { Confidencialidad como derecho del paciente }\end{array}$ & $\begin{array}{l}\text { Identificar los distintos tipos de } \\
\text { datos contenidos en la historia clínica } \\
\text { Identificar qué personas pueden acceder a ellos } \\
\text { Justificar las excepciones a la confidencialidad }\end{array}$ \\
\hline
\end{tabular}

de Evaluación de la Calidad habla de ética médica', se refiere, sin duda, a ética profesional, pero no a la bioética entendida como ética cívica, ni a sus contenidos específicos.

A modo de propuesta, se señalan en la tabla IV siete competencias que consideramos básicas en el área de la bioética, así como los conocimientos y habilidades que se requieren para alcanzar cada una de ellas.

\section{Desarrollo curricular}

El origen del programa curricular estructurado en dos ciclos es casi centenario [29]. Si bien es cierto que no ha sido fácil integrar coherentemente ambos niveles, o asumir en esta estructura curricular las competencias transversales (comunicación, aprendizaje a largo plazo, etc.), también lo es que este modelo sigue orientando 
muchos de los programas de nuestras facultades de medicina [30].

Los dos niveles de enseñanza, básico y clínico, rigen también para la bioética. Si un alumno no puede capacitarse en traumatología sin haber estudiado previamente anatomía, tampoco puede abordar problemas de valores en pediatría, medicina crítica o cuidados paliativos si previamente no ha adquirido unos conocimientos básicos en bioética.

En la etapa preclínica o básica se debe enseñar bioética básica. Se entregarán al alumno las herramientas elementales de la disciplina, a saber: en qué consiste, su origen, su relación con la ética cívica, qué es un juicio moral, los principios éticos de la relación clínica y su relación con los derechos constitucionales de los agentes implicados en ella, y también los procedimientos y metodología en ética. Se pretende introducir a los alumnos en la problemática ética inherente a la profesión sanitaria, y proporcionarles los contenidos teóricos fundamentales para acercarse a ella racional y críticamente. Debería impartirse en $2 .^{\circ}$ o 3 . $^{\text {er. }}$ cursos. Lo ideal es el 3 . $^{\text {er. }}$ curso, momento en el que el alumno está ubicado en la carrera, y comienza a contactar personalmente con la realidad clínica y con el enfermo.

En el período clínico, la bioética también debe serlo. Los alumnos deben adquirir los conocimientos y habilidades que les capaciten para resolver problemas específicos de la praxis clínica habitual de un médico general, no de un especialista. Tampoco tiene mucho sentido que todas y cada una de las asignaturas lleven adosado un correlato bioético, lo que, además de incrementar sin fundamento el programa curricular, no aporta nada positivo en la formación en bioética. Si bien el paciente con enfermedad de Alzheimer no es el mismo que el paciente con una enfermedad neurodegenerativa, sí lo es que el análisis de los problemas éticos que pueden plantear ambos requiere manejar los mismos o similares conceptos teóricos.

Por tanto, el contenido de la bioética clínica se puede estructurar alrededor de grandes paradigmas clínicos: problemas del origen de la vida, problemas del final de la vida y problemas específicos de algunas patologías, como el sida o la enfermedad mental, que requieren un abordaje específico. Puede obtenerse una sólida formación de los alumnos trabajando los problemas de estos bloques temáticos, y aplicando en ellos lo apren- dido en el nivel básico. Debería impartirse en $5 .^{\circ}$ curso, dejando para el último año lectivo, como ya se señaló, los contenidos específicos del profesionalismo.

\section{Conclusión}

Pese a sus peculiaridades, la enseñanza de la bioética en la medicina debería seguir los mismos planteamientos que el resto de las asignaturas. Definir competencias a desarrollar, hacerlo en los dos niveles (preclínico y clínico) y dentro del marco del aprendizaje por problemas es la mejor manera de enseñar al futuro profesional a tomar decisiones fundamentadas en el complejo mundo de los valores.

\section{Bibliografía}

1. Spaulding WB. Revitalising medical education: McMaster Medical School. The early years 1965-1974. Hamilton, ON: B.C. Decaer; 1991.

2. Emanuel E, Emanuel L. Cuatro modelos de la relación médico paciente. En Couceiro A, ed. Bioética para clínicos. Madrid: Triacastela; 1999. p.109-26.

3. Cortina A. La ética de la sociedad civil. Madrid: Anaya; 1994.

4. Hernando P. Los derechos de los pacientes: una cuestión de calidad. Rev Calidad Asistencial 2005; 20: 353-6.

5. Couceiro, A. Los niveles de la justicia sanitaria y la distribución de los recursos. An Sist Sanit Nav 2006; 29 (Supl 3): S61-74.

6. Pardell H. ¿Tiene sentido hablar de profesionalismo hoy? Educ Med 2003; 6: 63-80.

7. Borrell-Carrió F, Epstein RM, Pardell H. Profesionalidad y professionalism: fundamentos, contenidos, praxis y docencia. Med Clin (Barc) 2006; 127: 337-42.

8. ABIM Foundation, ACP-ASIM Foundation and European Federation of Internal Medicine. Medical professionalism in the new millenium: a physician charter. J Am Coll Surg 2003; 196: 115-8.

9. Couceiro A. Introducción a la bioética. En Rodés-Teixidor J, Guardia-Massó J, eds. Medicina interna. 2 ed. Barcelona: Masson, 2004; p. 16-21.

10. Gracia D. Planteamiento general de la bioética. En Gracia D, ed. Fundamentación y enseñanza de la Bioética. Bogotá: El Búho; 1998. p. 11-28. 
11. Pardell H. El nuevo profesionalismo médico. Una ideología expresada en conductas. En Oriol-Bosch A, Pardell H, eds. La profesión médica. Los retos del milenio. Barcelona: Fundación Medicina y Humanidades Médicas; 2005. p. 11-22.

12. Cortina A. Ética aplicada. En Cortina A, Martínez E, eds. Ética. Madrid: Akal; 1996. p. 151-84.

13. Cruess SR, Cruess RL. Professionalism must be thaught. BMJ 1997; 315: 1674-7.

14. Whitcomb ME. Fostering and evaluating professionalism in medical education. Acad Med 2002; 77: 473-4.

15. Gracia D. La bioética, una nueva disciplina académica. JANO 1987; 33: 309-13.

16. Baylis F, Downie J. Ethics education for Canadian medical students. Acad Med 1991; 66: 413-4.

17. Consensus statement by teachers of medical ethics and law in UK medical schools. Teaching medical ethics and law within medical education: a model for the UK core curriculum. J Med Ethics 1998; 24: 188-92.

18. Albanese MA, Mitchell S. Problem-based learning: a review of literature on its outcomes and implementation issues. Acad Med 1993; 68: 52-81.

19. Vernon DTA, Blake RL. Does problem-based learning work? A meta-analysis of evaluative research. Acad Med 1993; 68: 550-63.

20. Epstein RM, Hundert EM. Defining and assessing professional competence. JAMA 2002; 287: 226-35.

21. Smith SR, Dollase RH, Boss JA. Assesing student's per- formances in a competency-based curriculum. Acad Med 2003; 78: 97-107.

22. Culver C, Clouser K, Gert B, Brody H, Fletcher J, Jonsen A, et al. Basic curricular goals in medical ethics. N Engl J Med 1985; 312: 253-6.

23. Baldwin WC, Steven RD, Self DJ. Changes in moral reasoning during Medical School. Acad Med 1991; 66 (Suppl): S1-3.

24. Hundert EM. Characteristics of the informal curriculum and trainees' ethical choices. Acad Med 1996; 71: 624-8.

25. Self DJ, Daldwin DC, Wolinsky FD. Further exploration of the relationship between medical education and moral development. Camb Q Health Ethics 1996; 5: 444-9.

26. Institute for Internacional Medical Education. Global minimum essential requeriments in medical education. Educ Med 2003; 6 (Suppl 2).

27. Peinado JM. Competencias médicas. Educ Med 2005; 8 (Supl 2): S4-6.

28. González J, Wagenaar R. Tunning educational structures in Europe. Informe final. Deusto: Universidad de Deusto; 2003.

29. Flexner A. Medical education in the United States and Canada. New York: The Carnegie Foundation for the Advancement of Teaching; 1910. Bulletin 4.

30. Prat-Corominas J, Oriol-Bosch A. Nuevas orientaciones en los programas universitarios de preparación de profesionales médicos. En Oriol-Bosch A, Pardell H, eds. La profesión médica. Los retos del milenio. Barcelona: Fundación Medicina y Humanidades Médicas; 2005. p. 85-98. 